\title{
The Role of NADPH Oxidases in Cardiovascular Disease
}

\author{
Wen Zhang ${ }^{1}$, Juncai Bai', Juanjuan Tian¹, Lingxiao Jia $^{2}$ and Xiaoxu Zhou ${ }^{1 *}$
}

${ }^{1}$ Department of Cardiology, The First Affiliated Hospital of Harbin Medical University, Harbin, PR China

${ }^{2}$ Department of Clinical Laboratory, General Hospital of Daqing Oil Field, Daqing, PR China

\begin{abstract}
Cardiovascular disease is the high incidence of disease in the world and has the high rate of mortality and morbidity. Therefore, researching new mechanisms of cardiovascular disease has been received the widespread attention. A large number of studies have shown that oxidative stress is one of the main mechanisms of cardiovascular disease occurrence and development. Excessive reactive oxygen species cause an imbalance in the body of oxidation and anti-oxidation, which causes tissue damage. NADPH oxidase is the principal source of reactive oxygen species, thereby, studying clearly the role of NADPH oxidase in cardiovascular disease has important clinical significance for preventing and treating cardiovascular diseases via controlling oxidative stress. We summarized the newest studies on the relationship between NADPH Oxidases and cardiovascular diseases, which provide an idea that NADPH Oxidases play an important role in cardiovascular disease occurrence and development. This idea may illustrate the new cardiovascular disease pathogenesis and discovering new methods for cardiovascular diseases prevention and treatment.
\end{abstract}

Keywords: NADPH oxidases; Cardiovascular disease; Oxidative stress

\section{Introduction}

Cardiovascular disease (CVD) is one of the important diseases in the world and damages human health. The major risk factors of cardiovascular disease occurrence include age, gender, unhealthy diet, smoking, drinking, a high degree of mental stress and lack of exercise. A growing number of studies indicate that oxidative stress is an important factor for the occurrence and development of cardiovascular disease via vascular endothelial dysfunction, inflammation, apoptosis, cell migration, fibrosis, and angiogenesis in relation to vascular remodeling of hypertension $[1,2]$. Highly reactive molecules include reactive oxygen species (ROS), such as anion $\left(\mathrm{O}^{2}\right)$, hydroxyl free radical $\left(\mathrm{OH}^{-}\right)$and hydrogen peroxide $\left(\mathrm{H}_{2} \mathrm{O}_{2}\right)$, and reactive nitrogen free radicals (RNS) such as nitric oxide (NO), nitrogen dioxide $\left(\mathrm{NO}_{2}\right)$ and nitrite peroxide (ONOO $)^{-}$[3]. Researchers have found that the levels of reactive oxygen species are important for maintaining the normal physiological function of body and excessive levels of reactive oxygen species can cause body oxidative damage [4]. In mammalian cells, potential enzymatic sources of ROS include mitochondrial electron transport, lipoxygenase and cyclooxygenase enzymes of the arachidonic cascade, cytochrome p450 enzymes, xanthine oxidase, $\mathrm{NADH} / \mathrm{NADPH}$ oxidase, nitric oxide synthase (NOS), peroxidases and other hemoproteins. While the important sources of ROS, nicotinamide adenine dinucleotide phosphate (NADPH) oxidases play a central role [5] in this process. Therefore, it is important to research the role of NADPH oxidase in cardiovascular disease occurrence and development.

\section{NADPH oxidases}

NADPHoxidases werefirstly found in neutrophils and macrophages, and these two types of cells can produce a large number of ROS in the inflammatory response. The ROS was originally described as cytotoxic molecules and mainly protect the defense of the body by killing bacteria [3]. A number of studies indicated that NADPH oxidases located in the cytoplasm membrane of phagocytes. The NADPH oxidases, with cytochrome $\mathrm{C}$ and $\mathrm{FAD}$ group, are composed by two membranebound subunits 22phox and NOX2 (formerly known as gp91phox) which form cytochrome b558 as the catalytic core of this enzyme. Other important components of this enzyme are cytosolic subunits p40phox p47phox, p67phox and the small GTP-binding protein Rac. In addition to the above five main subunits, the activation process of enzyme needs the low molecular weight of $\mathrm{G}$ protein arcZ (sometimes racl) and the participation of raPIA [6-9]. A range of catalytic subunit NADPH oxidases were later found in different kinds of cells, such as NOX1, NOX2 (gp91phox), NOX3, NOX4, NOX5, DUOX1, DUOX2, which are belonged to NOX protein family. NOX can maintain physiological levels of $\mathrm{O}^{2-}$ and $\mathrm{H}_{2} \mathrm{O}_{2}$ with low expression and activity. When NOX is influenced by innate and acquired immune response or blood vessels injury in atherosclerosis, diabetes, obesity, high blood pressure and anoxia, the expression of NOX is increased and activated quickly to produce large quantities of ROS (Figure 1), which can mediate oxidative stress $[7,10,11]$. Studies have shown that ROS production is mediated by NADPH oxidases and ROS is an important signal molecule in the processes of controlling some key pathological activities including cell proliferation, amplification, migration, mutation, apoptosis, immune response, and signal transduction pathways [11]. The occurrence and development of diseases including cardiovascular disease, diabetes, obesity and cancer are associated with the ROS production mediated by NOX $[8,9,12]$. Lots of evidences showed that carotenoids, which were inversely associated with inflammatory cytokines, not only can take effect on anti-hypertension, but also delay the onset of wellestablished cardiovascular risk factors [13]. In recent years, researchers have found that the NADPH oxidases not only exist in phagocyte, but different NOX enzymes also constitutively expressed in vascular cells (such as endothelial cells, smooth muscle cells, fibroblasts, and pericytes), cardiomyocytes, and immune cells in circulating and tissue

${ }^{*}$ Corresponding author: Xiaoxu Zhou, Department of Cardiology, The Firs Affiliated Hospital of Harbin Medical University, Harbin, Heilongjiang, 150001, PR China, Tel: 086-0451-85555686; E-mail: xiaoxu_zhou@hotmail.com

Received March 06, 2016; Accepted April 25, 2016; Published May 03, 2016

Citation: Zhang W, Bai J, Tian J, Jia L, Zhou X (2016) The Role of NADPH Oxidases in Cardiovascular Disease. J Vasc Med Surg 4: 265. doi:10.4172/23296925.1000265

Copyright: ( 2016 Zhang W, et al. This is an open-access article distributed under the terms of the Creative Commons Attribution License, which permits unrestricted use, distribution, and reproduction in any medium, provided the original author and source are credited. 


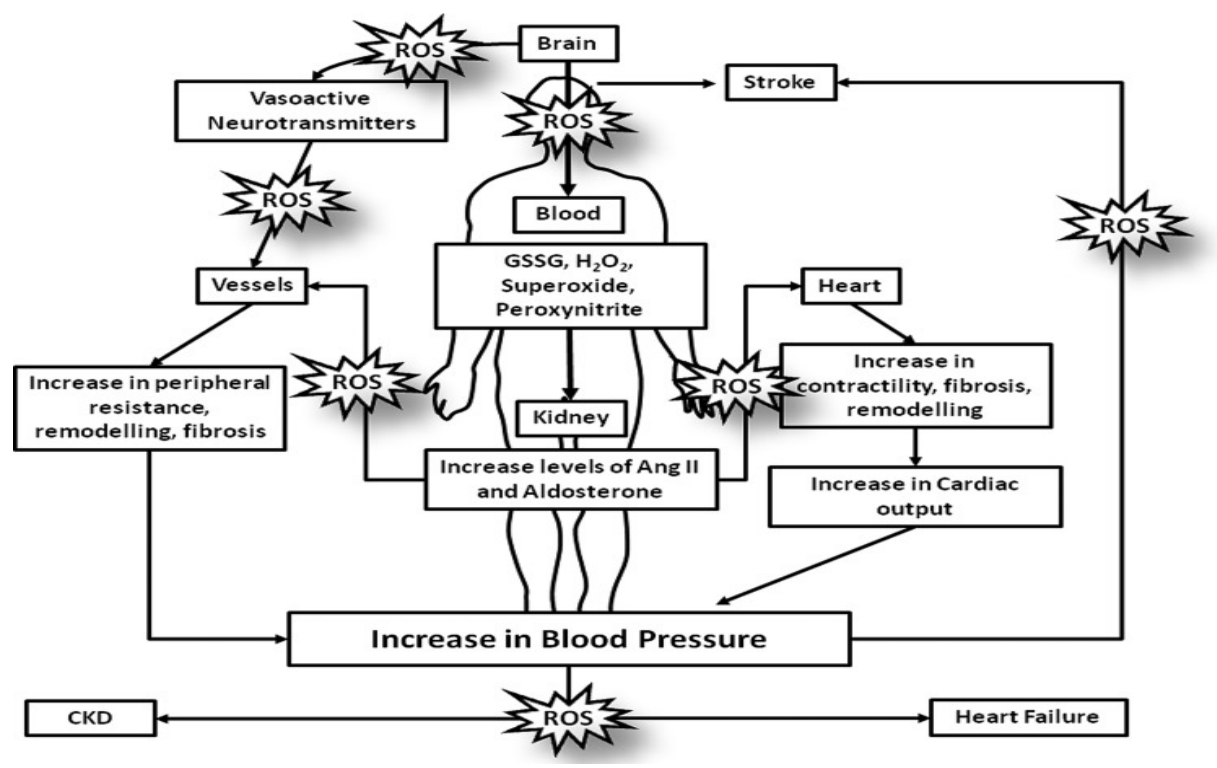

Figure 1: When NOX is influenced by innate and acquired immune response or blood vessels injury in atherosclerosis, diabetes, obesity, high blood pressure and anoxia, and the expression of NOX is increased and activated quickly to produce large quantities of ROS [14].

(including monocytes, macrophages dendritic cells and mast cells). In these cells, the NADPH oxidase is one of the important sources of producing reactive oxygen species $[7,8,11]$. Some studies have shown that the C242T polymorphism, the p22phox subunit of NADPH oxidase is related with coronary heart disease (CAD) and ischemic cerebrovascular disease (ICVD) and is an independent risk factor in high blood pressure, diabetes, hyperlipidemia and smoking $[8,14]$.

\section{The roles of NADPH oxidases in cardiovascular disease}

NOX1 and cardiovascular disease: NOX1 (Mox1, NOH1) has been identified as the first homologue of NOX2 and shares a $60 \%$ amino-acid sequence identity with NOX2. NOX1 expresses in endothelial, smooth muscle and adventitial cells of the vasculature. Lots of studies showed localization of NOX1 in cell membranes, particularly in the plasma membrane, by using recombinant NOX1 protein. The activity of NOX1 requires p22phox, NoxO1 (or possibly p47phox in some cases), NoxA1 and the small GTPase Rac. NOX1-dependent ROS generation has been shown to play a pivotal role in cell signaling, cell growth, angiogenesis and cell motility [15]. Some studies confirm that NOX1 plays an important role in the initial injury of coronary atherosclerosis. Barry - Lane and others found that total aortic atherosclerotic area was reduced in p47phox knockout mice, which suggested that NOX1 / NOX2 may be involved in atherogenesis [12]. Studies have also shown that the expression of NOX1 protein in myocardial cell after hypoxia was significantly increased, compared with the control group, and ROS produced in the cell also increased [16]. Via observing the expression of NOX1 and NOX4 in different time and location in the mice injured by focal cerebral ischemia reperfusion, researchers found that NOX1 expression in early ischemia increased obviously, which may be closely associated with early pathological damage process [17]. NOX1 mediating the expressions of vascular endothelial growth factor (VEGF) and the matrix metalloproteinase (MMPS) may increase the formation of reactive oxygen species, then NOX1 promotes angiogenesis and tumor formation [18]. NOX1 is also expressed in vascular smooth muscle cells, uterus, and glands. The main physiological function of NOX1 is involved in cell proliferation and angiogenesis and the occurrence and development of the hepatic fibrosis $[19,20]$. The role of NOX1 plays in the development of cardiovascular disease should be further researched.

NOX2 and cardiovascular disease: NOX2, originally called gp91phox, expresses in vascular smooth muscle cells (VSMCs), outer membrane fibroblast, endothelial cells and the fat cells around blood vessels. The main physiological functions of NOX2 are involved in immune defenses, oxidative stress and blood pressure regulation. There are six subunits of NOX2 including gp91phox, p22phox, p47phox, p67phox, p40phox and GTPase Rac1. Gp91phox and p22phox locate in the cytoplasm vacuoles and the plasma membrane, via membrane combination; form the cytochrome b558. The structure of this oxidase in vascular cells is similar to that in phagocytes. Nox organizer protein 1 (NoxO1) and Nox activator protein 1 (NoxA1) may have modest activating properties for Nox2 [21]. In human, the lack of NOX2 can strengthen endothelium-dependent flow-mediated vasodilatation and reduce vascular aging and oxidative stress [22]. Studies have shown that the mitochondria $\mathrm{O}^{2-}$ mediated by ATP sensitive channels depending on NADPH can activate the NOX2 in the cytoplasm, which promotes the development of oxidative stress production in endothelial cell and high blood pressure disease $[23,24]$. The myocardial infarction area is significantly reduced in the NOX1, NOX2 and NOX1/ NOX2 knockout mice, which suggested that NOX1 and NOX2 can mediate oxidative damage during reperfusion [25]. Other studies have reported that in the early stages of the atherosclerotic plaque development, NOX2 seems to play a key role in the progress of atherosclerosis occurrence and development [26].

NOX3 and cardiovascular disease: NOX3 is located in chromosome 6 and primarily expresses in the inner ear (including spiral ganglion, ear chamber and the epithelial cells of the cochlea), fetal kidney, spleen, bone, brain and Hep G2 in cancer cell line [10]. Previous studies have shown that cisplatin exposure is associated with reactive oxygen species (ROS) increase in the cochlea and cisplatin, a widely used chemotherapeutic agent, causes significant hearing loss. However, knockdown of NOX3 by pretreatment with siRNA prevented cisplatin 
ototoxicity, which is detected by examining preservation of hearing thresholds and inner ear sensory cells [27]. In addition, researchers have found that NOX3 is a very important susceptibility genes in the hearing loss induced by noise via a genome-wide association analysis [28]. Studies have also shown that NOX3 mRNA is related with the occurrence and development of gastric cancer [29]. However, whether NOX3 plays a role in the development of cardiovascular disease need to be investigated in the future.

NOX4 and cardiovascular disease: The NOX4 protein shares a $39 \%$ of amino acid fragment with NOX2. NOX4 was initially found in the kidney, therefore, NOX4 was named kidney oxide (KOX). Then, it was soon found in vascular wall (especially in vascular smooth muscle cells, fibroblasts and endothelial cells), heart, skeletal muscle and brain, but the expression of NOX4 mRNA in mononuclear cells is low. The main physiological function of NOX4 is regulation of the synthesis of erythropoietin and oxygen sensors. In some studies, researches have reported that NOX4 in kidney and smooth muscle cells may play an important role in stressing signal transduction. NOX4 can adjust the differentiation induced by the transforming growth factor beta (TGF- $\beta$ ), insulin signal transduction, transcriptional regulation and myocardial differentiation [12]. NOX4 mainly locates in the endoplasmic reticulum and nucleus, and the activity of NOX4 mainly depends on the levels of NOX4 and p22phox expressions. But, recent studies have not shown clearly which type of ROS is predominantly generated by NOX4. Unlike NOX1 and NOX2 mainly producing $\mathrm{O}^{2}$ , NOX4 mainly produce hydrogen peroxide $\left(\mathrm{H}_{2} \mathrm{O}_{2}\right)$. It is likely for a highly conserved histidine residue in the E-loop of NOX4 promoting rapid dis-mutation of $\mathrm{O}^{2-}$ [2]. NOX4 is increased after ischemia, which may be related with the process of pathological repairing after ischemia - reperfusion [17]. In addition, the up-regulation of NOX4 expression can cause endothelial cell damage mediated by ROS, which is associated with hypertension, hyperlipidemia, and ischemic diseases [30-33]. Active NOX4 also can increase the formation of ROS, ERK1/2 and JNK phosphorylation and collagen synthesis increase, which contribute to the process of myocardial fibrosis. Thus, myocardial remodeling is improved by reducing the myocardial oxidative stress and the myocardial fibrosis via NOX4 intervening [34]. In human coronary artery endothelial cells, oxidized low-density lipoprotein (oxLDL) can promote the generation of ROS by activating NOX4 [18]. In a recent study, a novel nuclear-localized NOX4 splice variant with a size of $28-\mathrm{kDa}$ protein, NOX4D, has been identified and NOX4D lacks putative transmembrane domains. Over expression of NOX4D can result in the increase of NADPH-dependent ROS production and phosphorylation of extracellular signal-regulated kinase $1 / 2$ and the nuclear transcription factor Elk-1 [35]. Therefore, NOX4D may take important pathophysiologic effects on the modulation of nuclear signaling and DNA damage [35]. The recent study about NOX4 also shows that NOX4 takes the effect on anti-atherosclerosis [36,37]. However, NOX4 expression is decreased in human abdominal aortic aneurysm (AAA), although NOX4 greatly promote the progress of oxidative stress occurrence in abdominal aortic aneurysm walls [38].

NOX5 and cardiovascular disease: NOX5 expresses in all embryonic organization and a weak expression of NOX5 is in the ovary, placenta, pancreas and human microvascular endothelial cells. NOX5 protein was found in the endoplasmic reticulum of human microvascular endothelial cells (HMEC-1) and the vascular wall $[10,35]$. HMEC- 1 cells expressed NOX5 $\beta$ and NOX5 $\delta$ as well as a variant lacking calcium-binding domain (NOX5S). NOX5 $\beta$ and NOX5S increase basal ROS levels. NOX5, different with the other NOX proteins, has a unique $\mathrm{N}$-terminus which encodes four calcium- binding EF hands. NOX5 conformation will be changed when the calcium-binding EF hands combined with $\mathrm{Ca}^{2+}$, which caused the intramolecular interaction in the catalysis between $\mathrm{N}$ - terminus and Cterminus, and then the free $\mathrm{Ca}^{2+}$ will promote the ROS production. Four splicing variants have been confirmed as NOX $5 \alpha$, NOX $5 \beta, N O X 5 \gamma$ and NOX $5 \delta$ [39]. NOX $5 \alpha$ was found in spleen, and NOX $5 \beta$ was found in testis whereas the cellular presences of NOX $5 \gamma$ and NOX $5 \delta$ have not been shown [40], in addition, NOX5 mRNA was found in the stomach and cardiac fibroblasts [7]. NOX5 also exists in vascular structures, especially endoplasmic reticulum of endothelial cells and vascular smooth muscle cells, which mainly produces superoxide and $\mathrm{H}_{2} \mathrm{O}_{2}$. Some studies have suggested that the different subtypes of NOX located in different positions during the development of atherosclerosis, and NOX5 is mainly expressed in human unstable atherosclerotic plaque [26]. In the coronary artery disease, levels of NOX5 protein and mRNA expression increase in human coronary artery, especially in the early stages of endothelial injury [12]. Another study indicated that NOX5S contributed to endothelial ROS generation, proliferation and angiogenesis. However, the role of NOX5 in vascular cells has not been fully elucidated $[7,41]$.

The roles of DUOX1 and DUOX2 in cardiovascular disease: DUOX1 and DUOX2 mainly exist in thyroid tissue and express lowly in the others tissues including salivary glands, bronchial, lung and prostate. The $\mathrm{H}_{2} \mathrm{O}_{2}$ expression mediated by DUOX2 can induce oxidative stress, which results in the intestinal tissue injury $[10,12,26]$. Enhanced reactive oxygen species production in allergic airways are well described and correlated with airway contractions increase, inflammatory cell infiltration, goblet cell metaplasia and mucus hyper secretion. DUOX activity is regulated by cytokines including IL-4 and IL-13, and DUOX-mediated $\mathrm{H}_{2} \mathrm{O}_{2}$ influences several important features of allergic asthma including mucin production, IL-8 secretion and wound healing [42]. In the thyroid gland, $\mathrm{H}_{2} \mathrm{O}_{2}$ mediated by DUOX2 is the important material of thyroid hormone biosynthesis. Several patients with the gene mutations of DUOX2 or its maturation factor, DUOXA2, have serious organic iodine machine function defect [43].

\section{Conclusion}

Oxidative stress, one of the important pathogenesis of cardiovascular disease, interacts with other pathogenesis directly or indirectly involved in the occurrence and development of the disease, such as hypertension, heart failure, atherosclerosis and myocardial injury [44]. In recent years, a large number of experimental studies found that NADPH oxidase is the main source of ROS in the blood vessel cells, however, the relationship between NOX and cardiovascular disease has not yet fully elucidated. We summarized the newest researches results about NOX and cardiovascular diseases and provide an idea that NOX play an important role, which may illustrate the new cardiovascular disease pathogenesis and discover new methods for cardiovascular diseases prevention and treatment. In clinic, there is still lack of effective antioxidant drug for inhibiting NADPH oxidase, thus, further studies about the role and mechanism of NADPH oxidase in heart vascular disease will be investigate.

\section{Acknowledgment}

This study was supported by grants from National Natural Science Foundation of China (Project No. 81300202) and Heilongiiang Province Foundation for the Returned Overseas Scholar (Project No. LC2012C34)

\section{References}

1. Cai H, Harrison DG (2000) Endothelial dysfunction in cardiovascular diseases: the role of oxidant stress. Circ Res 87: 840-844. 
2. Takac I, Schroder K, Zhang L, Lardy B, Anilkumar N, et al. (2011) The E-loop is involved in hydrogen peroxide formation by the NADPH oxidase Nox4. J Biol Chem 286: 13304-13313.

3. Segal AW (2005) How neutrophils kill microbes. Annu Rev Immunol 23: 197 223

4. Ma QL, Zhang GG, Peng J (2013) Vascular peroxidase 1: a novel enzyme in promoting oxidative stress in cardiovascular system. Trends Cardiovasc Med 23: $179-183$

5. Hristova M, Penev M (2014) Oxidative Stress and Cardiovascular Diseases. Trakia Journal of Sciences 3: 296-303.

6. Babior BM (1995) The respiratory burst oxidase. Curr Opin Hematol 2: 55-60.

7. BelAiba RS, Djordjevic T, Petry A, Diemer K, Bonello S, et al. (2007) NOX5 variants are functionally active in endothelial cells. Free Radical Biology \& Medicine 42: 446-459.

8. Han HX, Zhang C (2004) E242T polymorphism of NADPH oxidase p22phOX gene and ischemia cerebrovascular disease.

9. Lambeth JD, Cheng G, Arnold RS, Edens WA (2000) Novel homologs of gp91phox. Trends Biochem Sci 25: 459-461.

10. Li-li L, Sheng-song T (2008) Tissue distribution and physiological function of NOX family NADPH oxidases. International Journal of Pathology and Clinical Medicine 28: 19-23.

11. Manea SA, Constantin A, Manda G, Sasson S, Manea A (2015) Regulation of Nox enzymes expression in vascular pathophysiology: Focusing on transcription factors and epigenetic mechanisms. Redox Biol 5: 358-366.

12. Konior A, Schramm A, Czesnikiewicz-Guzik M, Tomasz J, Guzik TJ (2014) NADPH oxidases in vascular pathology. Antioxid Redox Signal 20: 2794-2814

13. Ciccone MM, Cortese F, Gesualdo M, Carbonara S, Zito A, et al. (2013) Dietary intake of carotenoids and their antioxidant and anti-inflammatory effects in cardiovascular care. Mediators Inflamm 2013: 782137.

14. Montezano AC, Touyz RM (2014) Reactive oxygen species, vascular Noxs and hypertension: focus on translational and clinical research. Antioxid Redox Signal 20: 164-182.

15. Arbiser JL, Petros J, Klafter R, Govindajaran B, McLaughlin ER, et al. (2002) Reactive oxygen generated by Nox1 triggers the angiogenic switch. Proc Nat Acad Sci USA 99: 715-720.

16. Lei W, Ju-xiang L, Kui H, Hao D, Zi-rong X, et al. (2009) Change of subunit of NADPH oxidation enzyme complex Nox- 1 protein in cardiocyte hypoxiareoxygenation injury and the role of cardiotrophin. Chinese Journal of pathophysiology 25: 2113-2117.

17. Chen-chen Z, Pei L, yu-ke T, Hui X (2013) Expressions of Nox1 and Nox4 in Mice after Focal Cerebral Ischemia and Reperfusion Injury. Neural Injury and Functional Reconstruction 8: 329-333.

18. Thum T, Borlak J (2004) Mechanistic role of cytochrome P450 monooxygenase in oxidized low-density lipoprotein-induced vascular injury: therapy through LOX-1 receptor antagonism? Circ Res 94: e1-13.

19. Shan-Shan $Y$, Xuan Zhu X (2014) Role of NADPH oxidase family members in promoting liver fibrosi. World Chinese Journal of Digestology 22: 2710-2715.

20. Paik YH, Iwaisako K, Seki E, Inokuchi S, Schnabl B, et al. (2011) The nicotinamide adenine dinucleotide phosphate oxidase (NOX) homologues NOX1 and NOX2/gp91(phox) mediate hepatic fibrosis in mice. Hepatology 53 : $1730-1741$.

21. Kawano M, Miyamoto K, Kaito $Y$, Sumimoto H, Tamura M (2012) Noxa1 as a moderate activator of Nox2-based NADPH oxidase. Arch Biochem Biophys 519: $1-7$

22. Violi F, Sanguigni V, Carnevale R, Plebani A, Rossi P, et al. (2009) Hereditary deficiency of gp91(phox) is associated with enhanced arterial dilatation: results of a multicenter study. Circulation 120: 1616-1622.

23. Nazarewicz RR, Dikalova AE, Bikineyeva A, Dikalov SI (2013) Nox2 as a potential target of mitochondrial superoxide and its role in endothelial oxidative stress. Am J Physiol Heart Circ Physiol 305: H1131-1140.

24. Dikalov SI, Nazarewicz RR, Bikineyeva A, Hilenski L, Lasse gue B, et al (2014) Nox2-induced production of mitochondrial superoxide in angiotensin II- mediated endothelial oxidative stress and hypertension. Antioxid Redox Signal 20: $281-294$

25. Braunersreuther V, Montecucco F, Asrih M, Pelli G, Galan K, et al. (2013) Role of NADPH oxidase isoforms NOX, NOX2 and NOX4 in myocardial ischemia/ reperfusion injury. J Mol Cell Cardiol 64: 99-107.

26. Guzik TJ, Chen W, Gongora MC, Guzik B, Lob HE, et al. (2008) Calciumdependent NOX5 nicotinamide adenine dinucleotide phosphate oxidase contributes to vascular oxidative stress in human coronary artery disease. J Am Coll Cardiol 52: 1803-1809.

27. Rybak LP, Mukherjea D, Jajoo S, Kaur T, Ramkumar V (2012) siRNA-mediated knock-down of NOX3: therapy for hearing loss? Cell Mol Life Sci 69: 24292434

28. Lavinsky J, Crow AL, Pan C, Wang J, Aaron K, et al. (2015) Genome-wide association study identifies nox 3 as a critical gene for susceptibility to noiseinduced hearing loss. PLoS Genet 11: e1005094.

29. Hong-qian L, Yong-chao C, Ya-lin J (2015) Expression of NADPH Oxidase NOX2 and NOX3 mRNA in Human Gastric Cancer. J Henan Univ Sci Tech 33: $1-3$

30. Xi H, Akishita M, Nagai K, Yu W, Hasegawa H, et al. (2007) Potent free radical scavenger, edaravone, suppresses oxidative stress-induced endothelial damage and early atherosclerosis. Atherosclerosis 191: 281-289.

31. van den Oever IA, Raterman HG, Nurmohamed MT, Simsek S (2010) Endothelial dysfunction, inflammation, and apoptosis in diabetes mellitus. Mediators Inflamm 2010: 792393

32. Vendrov AE, Vendrov KC, Smith A, Yuan J, Sumida A, et al. (2015) NOX4 NADPH oxidase-dependent mitochondrial oxidative stress in aging-associated cardiovascular disease. Curr Hypertens Rev 11: 132-142.

33. Gough DR, Cotter TG (2011) Hydrogen peroxide: a Jekyll and Hyde signalling molecule. Cell Death Dis 2: e213.

34. Menglong W, Jianfang L, Menglin L, Ying F, Wenhui Y, et al. (2015) Expression of NADPH oxidase in heart failure patients and its relationship with myocardium fibrosis. Chin J Diffic and ComplCas 14: 675-678.

35. Anilkumar N, Jose GS, Sawyer I, Santos CX, Sand C, et al. (2013) A 28-kDa splice variant of NADPH oxidase-4 is nuclear-localized and involved in redox signaling in vascular cells. Arterioscler Thromb Vasc Biol 33: e104-e112.

36. Sinha N, Dabla PK (2015). Oxidative stress and antioxidants in hypertension-a currentreview. Current Hypotension Reviews 11: 132-142.

37. Schürmann C, Rezende F, Kruse C, Yasar Y, Lowe O, et al. (2015) The NADPH oxidase Nox4 has anti-atherosclerotic functions. Eur Heart J 36: 3447-3456.

38. Guzik B, Sagan A, Ludew D, Mrowiecki W, Chwala M, et al. (2013) Mechanisms of oxidative stress in human aortic aneurysms--association with clinical risk factors for atherosclerosis and disease severity. Int J Cardiol 168: 2389-2396.

39. Cheng G, Cao Z, Xu X, van Meir EG, Lambeth JD (2001) Homologs of gp91phox: cloning and tissue expression of Nox, Nox, and Nox5. Gene 269 131-140.

40. Banfi B, Molnar G, Maturana A, Steger K, Hegedus B, et al. (2001) A Ca(2+) activated NADPH oxidase in testis, spleen, and lymph nodes. J Biol Chem 276 37594-37601.

41. Hahn NE, Meischl C, Kawahara T, Musters RJ, Verhoef VM, et al. (2012) NOX5 expression is increased in intramyocardial blood vessels and cardiomyocytes after acute myocardial infarction in humans. Am J Pathol 180: 2222-2229.

42. Chang S, Linderholm A, Franzi L, Kenyon N, Grasberger H, et al. (2013) Dual oxidase regulates neutrophil recruitment in allergic airways. Free Radic Biol Med 65: 38-46

43. Carvalho DP, Dupuy C (2013) Role of the NADPH Oxidases DUOX and NOX4 in Thyroid Oxidative Stress. Eur Thyroid J 2: 160-167.

44. Cardoso-Weide LC, Cardoso-Penha RC, Costa MW, Ferreira AC, Carvalho DP, et al. (2015) DuOx2 promoter regulation by hormones, transcriptional factors and the coactivator TAZ. Eur Thyroid J 4: 6-13. 